\title{
Robust Sliding Control of SEIR Epidemic Models
}

\author{
Asier Ibeas, ${ }^{1}$ Manuel de la Sen, ${ }^{2}$ and Santiago Alonso-Quesada ${ }^{2}$ \\ ${ }^{1}$ Departament de Telecomunicació i Enginyeria de Sistemes, School of Engineering, Universitat Autònoma de Barcelona, \\ 08193 Bellaterra, Barcelona, Spain \\ ${ }^{2}$ Department of Electricity and Electronics, Faculty of Science and Technology, University of the Basque Country, \\ P.O.Box 644, 48080 Bilbao, Spain \\ Correspondence should be addressed to Asier Ibeas; asier.ibeas@uab.cat
}

Received 12 November 2013; Revised 14 January 2014; Accepted 15 January 2014; Published 13 March 2014

Academic Editor: Xinzhu Meng

Copyright (C) 2014 Asier Ibeas et al. This is an open access article distributed under the Creative Commons Attribution License, which permits unrestricted use, distribution, and reproduction in any medium, provided the original work is properly cited.

This paper is aimed at designing a robust vaccination strategy capable of eradicating an infectious disease from a population regardless of the potential uncertainty in the parameters defining the disease. For this purpose, a control theoretic approach based on a sliding-mode control law is used. Initially, the controller is designed assuming certain knowledge of an upper-bound of the uncertainty signal. Afterwards, this condition is removed while an adaptive sliding control system is designed. The closed-loop properties are proved mathematically in the nonadaptive and adaptive cases. Furthermore, the usual sign function appearing in the sliding-mode control is substituted by the saturation function in order to prevent chattering. In addition, the properties achieved by the closed-loop system under this variation are also stated and proved analytically. The closed-loop system is able to attain the control objective regardless of the parametric uncertainties of the model and the lack of a priori knowledge on the system.

\section{Introduction}

Mathematical models have become an important tool in analyzing the causes, dynamics, and spread of epidemics. Thus, its study is crucial in order to obtain valuable knowledge of the underlying aspects of the disease. Furthermore, the analysis of mathematical models describing epidemics spreading allows us to make decisions regarding the best vaccination policies, quarantine application, and so on. In this way, a large number of mathematical models have been proposed in the literature (see [1-6] for more information on mathematical models). In addition, many specific features regarding these models have been studied in many works such as the presence of bifurcations $[5,7,8]$, the existence of equilibrium points and oscillatory behavior [9], and the presence of waves $[6,10]$ to cite just a few. However, model stability has been by far the most important property to be studied, $[8,11-15]$. One of the most fundamental issues when studying stability is the determination of the so-called reproduction number of the model, $R_{0}$. This number is defined as the expected number of secondary cases generated by one typical primary case in an entirely susceptible and sufficiently large population [16]. The reproduction number usually determines the stability of the equilibrium points when they exist. Typically, a reproduction number $R_{0}<$ 1 makes the disease-free equilibrium point asymptotically stable while $R_{0}>1$ makes the endemic equilibrium point locally asymptotically stable. In this way, for $R_{0}<1$ the epidemic is eradicated by itself while for $R_{0}>1$ there are always a number of infectious and infective people among the population. In this case, vaccination becomes an essential tool to eradicate the illness.

Control theory has recently emerged as one of the proposed approaches to deal with the design of vaccination campaigns $[7,17]$. Thus, different types of vaccination laws based on control theory have appeared in the literature during the last years such as the state-feedback control [17], feedback linearization [7, 18], and observer-based control, [19]. In addition, pulse vaccination has also gained much attention during the last decade $[9,20,21]$ mainly after its successful application in the eradication of poliomyelitis and measles across Central and South America [20]. Nevertheless, all 
these works assume the parameters defining the epidemics, such as the birth and mortality rates or the incidence rate to be perfectly known. This is a rather unrealistic situation since the estimation of such parameters from experimental data may lead to inaccurate values (i.e., values with an amount of uncertainty). As a consequence, the vaccination law is miscalculated and its application to the actual system may prevent the illness to be eradicated, as desired. Hence, the objective of this paper is to design a robust vaccination law capable of overcoming the potential mismatch between the nominal values used to design the control law and the actual parameters of the system. Therefore, the main novelty of the paper with respect to previous works is to consider the epidemics spreading control in the absence of complete knowledge on the system.

There are several strategies to design robust controllers such as $H_{\infty}$, fuzzy, or neural network based techniques [22-24]. Among them, sliding-mode control is one of the most well-known and extended techniques due to its simple design process and superior obtained results. Thus, it has successfully been applied to robotic manipulators [25], vehicle dynamics [26, 27], chemical engineering [28-30], and electrical systems $[31,32]$ to cite just a few. However, it has only been superficially applied before to the design of a vaccination law in the context of epidemic models [33], which is the main purpose of this work. In this way, this paper presents the design of a sliding-mode robust controller for a SEIR epidemic model. The designed vaccination law is able to make the total population become immune through time regardless of the potential inaccuracy in the knowledge of the parameters of the model. Typically, slidingmode controllers need to assume an a priori knowledge of the bounds of the uncertainties. This assumption may also appear somewhat unrealistic in epidemic models. Hence, the introduced sliding-mode controller is then enhanced with an adaptive mechanism to adapt online the value of the sliding gain. At the end, the whole system works in an autonomous manner without requiring difficult a priori estimations of the parameters or of their bounds while guaranteeing the epidemics eradication. Moreover, mathematical proofs of all the results stated in the paper are developed. These include the stability proof of the nonadaptive and adaptive sliding-mode controllers and a discussion on the effect of substituting the sign function by a saturation one in the control law. These proofs are far from being trivial and have not been considered with detail in previous works [33].

The paper is organized as follows. Section 2 introduces the epidemic model to be considered. Section 3 presents the sliding-mode control law and its properties. In this way, two different approaches will be proposed. The first one assumes the knowledge of an upper-bound of the potential mismatch between the nominal and the actual parameters of the model while the second approach removes this assumption by using an adaptive mechanism. Finally, Section 4 contains some simulation examples illustrating the theoretical results introduced in Section 3 while conclusions end the paper.

\section{Model Description}

Consider the SEIR epidemic model described by the following equations [17]:

$$
\begin{aligned}
\dot{S}(t)= & -\mu S(t)+\omega R(t) \\
& -\varphi(S, E, I, R)+\nu N(t)-N(t) V(t), \\
\dot{E}(t)= & \varphi(S, E, I, R)-(\mu+\sigma) E(t), \\
\dot{I}(t)= & -(\mu+\gamma) I(t)+\sigma E(t), \\
\dot{R}(t)= & -(\mu+\omega) R(t)+\gamma I(t)+N(t) V(t),
\end{aligned}
$$

where $S(t), E(t), I(t)$, and $R(t)$ denote the subpopulations of susceptible, infected, infectious, and immune population, respectively, and define the state vector $x^{T}=$ [ $\left.\begin{array}{llll}S & E & I & R\end{array}\right] . N(t)$ denotes the total population at each time $t$ (i.e., $N(t)=S(t)+E(t)+I(t)+R(t)), \mu$ is the rate of deaths from causes unrelated to the infection, $v$ denotes the birth rate, and $\omega$ is the rate of losing immunity. The typically nonlinear function $\varphi(S, E, I, R)$ is referred to as the disease incidence rate. When $\varphi(S, E, I, R)=\beta S(t) I(t)$, it is said to be the bilinear incidence rate; when $\varphi(S, E, I, R)=$ $\beta(S(t) I(t) / N(t))$, it is said to be the standard incidence rate and when $\varphi(S, E, I, R)=\beta S(t) I(t) /(1+\alpha S(t))$ or $\varphi(S, E, I, R)=\beta S(t) I(t) /(1+\alpha I(t))$, it is said to be the saturated incidence rate, where $\beta$ is the transmission constant (with, for instance, the total number of new infections per unit of time at time $t$ being $\beta(S(t) I(t) / N(t))$ for the standard incidence rate) and $\alpha$ is the saturation coefficient. $\sigma^{-1}$ and $\gamma^{-1}$ are, respectively, the average durations of the latent and infective periods. All the above parameters are assumed to be positive so as to represent a real situation. These parameters can be arranged into a single vector $\theta$ as follows:

$$
\theta^{T}=[\mu \omega \nu \sigma \gamma \beta \alpha]
$$

in such a way that the SEIR model (1)-(4) can be written as

$$
\dot{x}(t)=F(x(t), \theta)
$$

highlighting its explicit dependence on the parameters, $\theta$. The total population dynamics at time $t$ can be calculated by summing up all the equations (1)-(4), leading to

$$
\dot{N}(t)=(\nu-\mu) N(t) \text {. }
$$

It is worth noticing that the vaccination function $V(t)$ represents the fraction of the total population to be vaccinated at each time instant, $t$. Thus, $V(t) \in[0,1]$ for all $t \geq 0$.

The objective of this work is to design a vaccination law $V(t)$ capable of achieving $R(t) \rightarrow N(t)$ as $t \rightarrow \infty$ when the exact values of the parameters in $\theta$ are unknown, that is, when a nominal set of parameters $\hat{\theta}$ is used to define $V(t)$ instead of the actual ones (contained in $\theta$ ). A sliding-mode control is designed in the next section to achieve this objective. 


\section{Robust Sliding Control Design}

This section contains the design of a robust sliding-mode control for the epidemic model (1)-(4) so that all the population becomes immune asymptotically. Thus, define the tracking error as

$$
e(t)=R(t)-N_{\text {ref }}(t),
$$

where $R(t)$ denotes the number of immune population at each time while $N_{\text {ref }}(t)$ is a tracking reference signal satisfying

$$
\begin{aligned}
N_{\text {ref }}(0) & =R(0), \\
\left(N_{\text {ref }}(t)-N(t)\right) & \longrightarrow 0, \quad t \longrightarrow \infty .
\end{aligned}
$$

Equation (9) specifies the fact that, initially, the tracking error (8) is zero, $e(0)=0$. On the other hand, (10) states the fact that the reference signal tends to be the total population asymptotically. The condition $e(t)=0$ for all $t$ means that all the population tends to be immune asymptotically according to the shape imposed by the reference signal $N_{\text {ref }}(t)$. This signal can be selected as desired by the designer while satisfying (9) and (10). The proposed reference signal used in the sequel for theoretical and simulation purposes is chosen as

$$
N_{\text {ref }}(t)=(R(0)-N(0)) e^{-\epsilon t}+N(t),
$$

where the parameter $\epsilon>0$ controls the rate at which the reference signal converges to the total population. The control objective is to attain $e(t)=0$ for all $t \geq 0$ regardless of the potential mismatch in the system's parameters. For this purpose, the following sliding variable $\Phi(t)$ with integral term is considered:

$$
\Phi(t)=e(t)+\lambda \int_{0}^{t} e(\tau) d \tau,
$$

where $\lambda$ is a constant positive gain. The sliding surface guaranteeing the control objective is given by

$$
\Phi(t)=e(t)+\lambda \int_{0}^{t} e(\tau) d \tau=0 .
$$

The control law is now designed in a two-step procedure. Firstly, an equivalent control $V_{\mathrm{eq}}(t)$ is calculated in such a way that the sliding surface could be reached if the actual parameters of the model were known. For this, let us obtain the derivative of the sliding surface (12):

$$
\begin{aligned}
\dot{\Phi}(t)= & \dot{e}(t)+\lambda e(t) \\
= & \dot{R}(t)-\dot{N}_{\text {ref }}(t)+\lambda R(t)-\lambda N_{\text {ref }}(t) \\
= & -(\mu+\omega) R(t)+\gamma I(t)+N(t) V(t)-(\nu-\mu) N(t) \\
& +\epsilon e^{-\epsilon t}(R(0)-N(0))+\lambda R(t)-\lambda N(t) \\
& -\lambda e^{-\epsilon t}(R(0)-N(0)) .
\end{aligned}
$$

For the sake of simplicity, take $\epsilon=\lambda$ and all the terms associated with initial conditions in (14) will vanish. Let us equate now (14) to zero to obtain the equivalent control:

$$
\begin{aligned}
& -(\mu+\omega) R(t)+\gamma I(t)+N(t) V(t) \\
& -(\nu-\mu) N(t)+\lambda R(t)-\lambda N(t)=0 .
\end{aligned}
$$

The equivalent control is given by the solution of (15) with respect to $V(t)$ :

$$
\begin{aligned}
V_{\mathrm{eq}}(t)=\frac{1}{N(t)} & {[(\lambda+\nu-\mu) N(t)} \\
& +(\mu+\omega-\lambda) R(t)-\gamma I(t)] .
\end{aligned}
$$

However, since the actual parameters of the system, $\theta$, are not known, we have to use the nominal ones, $\hat{\theta}$, to define the equivalent control (16) for practical use. In this way, define

$$
\begin{array}{ll}
\delta=\lambda+\nu-\mu, & \widehat{\delta}=\lambda+\widehat{v}-\widehat{\mu}, \\
\rho=\mu+\omega-\lambda, & \widehat{\rho}=\widehat{\mu}+\widehat{\omega}-\lambda,
\end{array}
$$

and the equivalent control law takes the form

$$
V_{\mathrm{eq}}(t)=\frac{1}{N(t)}[\widehat{\delta} N(t)+\widehat{\rho} R(t)-\widehat{\gamma} I(t)],
$$

where the nominal parameters have already been substituted. The application of the equivalent control (18) to the system (1)-(4) may not make it attain the control objective due to the mismatch between the actual and the nominal parameters. Hence, the equivalent control (18) is augmented in the following nonlinear way so as to provide a robust behavior with respect to uncertainties:

$$
V(t)=V_{\mathrm{eq}}(t)-\frac{g(x, t)}{N(t)} \operatorname{sgn}(\Phi(t)),
$$

where $\operatorname{sgn}(\cdot)$ is the sign function defined as

$$
\operatorname{sgn}(x)=\left\{\begin{array}{lll}
1 & \text { if } x>0 \\
0 & \text { if } x=0 \\
-1 & \text { if } & x<0
\end{array}\right.
$$

and $g(x, t)$ is a switching gain to be specified in order to be able to overcome the uncertainties in the parameters. Thus, the augmented control law (19) allows guaranteeing the convergence to zero of the tracking error (8) as it is proved in the sequel. Note that the enhanced control law (19) is nonlinear due to the presence of the sign function. Therefore, the closed-loop system obtained by applying (19) to the system of (1)-(4) becomes nonlinear as well. The switching gain is defined based on the following assumptions.

Assumption 1. There exists a state-dependent function $b(x, t)$ such that the following upper-bounding holds:

$$
|(\widehat{\delta}-\delta) N(t)+(\widehat{\rho}-\rho) R(t)-(\widehat{\gamma}-\gamma) I(t)| \leq b(x, t)
$$

for all $t \geq 0$. 
Assumption 2. The upper-bounding function $b(x, t)$ is known.

Assumption 3. The switching gain $g(x, t)$ is selected as

$$
g(x, t)=b(x, t)+\eta
$$

with $\eta>0$ arbitrary.

Assumption 1 is related to the maximum amount of uncertainty caused by the mismatch in the parameters. Note that the function $b(x, t)$ always exists since the model is parameterized by certain parameter values despite being unknown. Furthermore, Assumption 1 has also a sound epidemiological interpretation. In order to present it clearly, we can rewrite (21) as

$$
\begin{aligned}
\mid \widehat{\delta} N(t) & -\delta N(t)+\hat{\rho} R(t)-\rho R(t)-\widehat{\gamma} I(t)+\gamma I(t) \mid \\
= & \left|N(t) V_{\text {eq }}(t)-N(t) V_{\text {eq,true }}(t)\right| \\
= & N(t)\left|V_{\text {eq }}(t)-V_{\text {eq,true }}(t)\right| \leq b(x, t),
\end{aligned}
$$

where $V_{\text {eq }}(t)$ is the equivalent control law given by (18) and expressed in terms of the nominal values $\widehat{\theta}$, while $V_{\text {eq, true }}(t)$ denotes the equivalent control when the actual parameters are known. Therefore, Assumption 1 states the fact that the absolute value of the difference between the applied vaccination control functions when the parameters are known and when they are unknown is upper-bounded by a certain function. Hence, the assumption is directly related to the error made in the number of vaccines applied due to the parameters mismatch. Assumption 2 states the fact that the upper-bounding is effectively known. This is a typical assumption in sliding-mode control systems [34]. However, this assumption does not limit the applicability of the control since the upper-bound (21) could be calculated from lower- and upper-bounds in the system's parameters. Finally, Assumption 3 defines how the sliding gain $g(x, t)$ should be selected. Furthermore, Assumption 2 will be weakened in Section 3.1, where the switching gain $g(x, t)$ is adapted online removing any a priori knowledge on the parameters of the system or on their bounds. Now, the following theorem can be proved.

Theorem 4. Consider the SEIR epidemic model (1)-(4) with the control law (19). Thus, if Assumptions 1, 2, and 3 hold, then the tracking error $e(t)$ vanishes asymptotically.

Proof. The proof is carried out by using Lyapunov's stability theorem. Hence, consider the Lyapunov candidate function:

$$
L(t)=\frac{1}{2} \Phi(t)^{2}
$$

Its time-derivative is calculated as

$$
\begin{aligned}
\dot{L}(t)= & \Phi(t) \dot{\Phi}(t) \\
= & \Phi(t)[\dot{e}(t)+\lambda e(t)] \\
= & \Phi(t)[-\rho R(t)+\gamma I(t)+N(t) V(t)-\delta N(t)] \\
= & \Phi(t)[(\widehat{\rho}-\rho) R(t)+(\gamma-\widehat{\gamma}) I(t) \\
& \quad+(\widehat{\delta}-\delta) N(t)-g(x, t) \operatorname{sgn}(\Phi)] \\
& \leq \Phi(t)[b(x, t)-g(x, t) \operatorname{sgn}(\Phi)] \\
= & \Phi(t)[b(x, t)-b(x, t) \operatorname{sgn}(\Phi)-\eta \operatorname{sgn}(\Phi)] \\
\leq & -\eta|\Phi(t)| \leq 0 .
\end{aligned}
$$

Notice that Assumptions 1, 2, and 3 have been used to prove that the time-derivative is always negative definite. Hence, $L(t)$ is positive definite and radially unbounded while $\dot{L}(t)$ is negative definite. Thus, according to Lyapunov's direct method, the equilibrium point at the origin $\Phi(t)=$ 0 is globally asymptotically stable and, therefore, $L(t)$ tends to zero as time tends to infinity. Moreover, all trajectories starting out the sliding surface $\Phi=0$ must reach it in finite time and then will remain on this surface. This fact can be directly verified from (31) since

$$
\frac{1}{2} \frac{d}{d t} \Phi(t)^{2}=\frac{1}{2} \frac{d}{d t}|\Phi(t)|^{2} \leq-\eta|\Phi(t)|, \quad \Phi(0) \neq 0
$$

implies $|\Phi(t)| \leq|\Phi(0)|-\eta t$. Therefore, for a finite time $t s \leq$ $|\Phi(0)| / \eta$, the sliding surface is reached. Then, $L(t)=0$ and $\dot{L}(t)=0$ for $t \geq t$ s deducing that the system's trajectories remain on the surface. When this condition is met, the dynamic behaviour of the tracking error (12) is given by

$$
\dot{e}(t)+\lambda e(t)=0 .
$$

Thus, the tracking error tends to zero exponentially and the control objective is achieved regardless of the mismatch in the parameters values. In this way, $\left(R(t)-N_{\text {ref }}(t)\right) \rightarrow$ $0,(R(t)-N(t)) \rightarrow 0$ as $t \rightarrow \infty$ and all the population tends to be immune, eradicating the illness.

Therefore, this theorem guarantees the eradication of the illness when the main parameters of the disease are not known since it calculates an appropriate number of vaccines in this scenario. In this way, it provides authorities with effective tools to control the epidemic spreading from the very beginning in the absence of complete knowledge of the problem.

Remark 5. Note that the particular structure of the incidence rate $\varphi(S, E, I, R)$ does not appear in the control law (19). Thus, the proposed robust controller can be applied as it is to any model with any incidence rate. Hence, it has a wide range of applicability. 
Remark 6. On the other hand, one of the drawbacks appearing in this approach is that an upper-bounding function $b(x, t)$ must be known (Assumption 2) since the switching gain is defined based on its value (Assumption 3). In some cases this information may not be known beforehand which complicates the applicability of the proposed control. Therefore, in the next subsection the switching gain is adapted through time in order to avoid the required a priori knowledge on the upper-bound of the signals.

Remark 7. The rate at which the reference signal, $N_{\text {ref }}(t)$, converges to the total population, $N(t)$, is given by $\epsilon$. This value is made equal to $\lambda$ in (15) in order to simplify the derivation of the control law. Thus, the convergence rate influences the control law (19) through $\lambda$. This means that the control law depends on the desired convergence rate implying that a different convergence rate requires a different vaccination law. This fact will be illustrated in Section 4 through simulation examples. Moreover, when $\lambda \neq \epsilon$, the general control law can be obtained from (14) straightforwardly.

3.1. Adaptation of the Sliding Gain. In this section, the switching gain $g(x, t)$ in (19) is automatically adapted online with the aim of removing the beforehand knowledge on the upper-bound of the mismatch signal (Assumption 2). However, Assumption 1 concerning the existence of such upper-bound is still applied but it is not explicitly used during the controller design process. Furthermore, the following Assumption 8, extending Assumption 1, is used in the sequel to just prove the stability properties of the scheme.

Assumption 8. There exists a finite, potentially unknown, positive constant $\bar{b}$ such that

$$
\bar{b} \geq b(x, t)
$$

for all $t \geq 0$.

Notice that Assumption 1 states the fact that the upper-bounding may be a state-depending function while Assumption 8 extends this assumption requiring the function $b(x, t)$ to be upper-bounded by a constant. From a biological perspective, this assumption implies that the absolute error between the nominally calculated and truly required vaccination laws (i.e., a measure of the vaccination control discrepancy due to modeling errors) is upperbounded by a constant. However, it is worth noting that this upper-bounding may be unknown since it is not explicitly used in the design of the controller but only in the proof of stability.

There are two ways of adapting the switching gain. The first one consists in starting with a very high value and then reducing it until it arrives to an appropriate tuning [25]. The other one consists in starting with a value of zero and then increasing it until the sliding condition is met [31]. This second approach is used in this subsection to perform the adaptation of the sliding gain. For this, the control equation (19) is changed to

$$
V(t)=V_{\mathrm{eq}}(t)-\frac{\widehat{g}(t)}{N(t)} \Gamma \operatorname{sgn}(\Phi(t)),
$$

where $\hat{g}(t)$ denotes the time-varying switching gain and $\Gamma \geq$ 1 is a constant defined by the designer. The switching gain is updated by

$$
\frac{d \widehat{g}(t)}{d t}=\Gamma \Phi(t) \operatorname{sgn}(\Phi(t))=\Gamma|\Phi(t)|, \quad \widehat{g}(0)=0 .
$$

Note that (36) defines a potentially diverging equation since the derivative of $\hat{g}$ is always nonnegative through time. The key issue to guarantee the stability of the closed-loop is that, although it is not known, the constant upper-bound given by Assumption 8 exists. Thus, the switching gain increases until it reaches a sufficiently high value for which upper-bounding (34) is achieved. Then, the system converges to the sliding surface and the sliding condition is met, making $\Phi=0$ and stopping the increase in $\hat{g}$. The combined working of these mechanisms makes the entire system stable as the following theorem states.

Theorem 9. Consider the SEIR epidemic model (1)-(4) with control law (35)-(36). Thus, if Assumptions 1 and 8 hold for an unknown but finite constant upper-bound $\bar{b}$ and $\Gamma \geq 1$, then the tracking error $e(t)$ vanishes asymptotically.

Proof. Firstly, notice that since Assumption 8 holds there exists an unknown but bounded constant $g$ satisfying $g \geq$ $\bar{b}+\eta$ for any constant $\eta>0$. Now, the proof is again based on Lyapunov's stability theorem. Define the following Lyapunov candidate function [31]:

$$
L(t)=\frac{1}{2} \Phi(t)^{2}+\frac{1}{2} \widetilde{g}(t)^{2},
$$

where $\widetilde{g}(t)=\widehat{g}(t)-g$ is the difference between the actual value of $\widehat{g}(t)$ at each time and the constant unknown upperbound $g$. The time-derivative of (37) is calculated as

$$
\begin{aligned}
\dot{L}= & \Phi(t) \dot{\Phi}+\widetilde{g} \dot{\hat{g}} \\
= & \Phi(t)[(\widehat{\rho}-\rho) R(t)+(\gamma-\widehat{\gamma}) I(t) \\
& \quad+(\widehat{\delta}-\delta) N(t)-\widehat{g} \Gamma \operatorname{sgn}(\Phi)]+\widetilde{g} \Gamma|\Phi(t)| \\
& \leq \Phi(t)[b(x, t)-\widehat{g} \Gamma \operatorname{sgn}(\Phi)]+(\widehat{g}-g) \Gamma|\Phi(t)| \\
= & b(x, t) \Phi(t)-\widehat{g} \Gamma|\Phi(t)|-g \Gamma|\Phi(t)|+\widehat{g} \Gamma|\Phi(t)| \\
& =b(x, t) \Phi(t)-g \Gamma|\Phi(t)| \\
\leq & |b(x, t)| \Phi(t)-\bar{b} \Gamma|\Phi(t)|-\eta \Gamma|\Phi(t)| \\
\leq & -\eta \Gamma|\Phi(t)| \leq 0 .
\end{aligned}
$$

Thus, $L(t)$ is a positive definite radially unbounded function in the variables $[\Phi \tilde{g}]$ while $\dot{L}(t)$ is negative semidefinite 
function. Hence, according to Lyapunov's direct method, the equilibrium point at the origin is globally stable. Notice that we cannot conclude the asymptotic stability of the system since the derivative of the Lyapunov function is only negative semidefinite. However, a contradiction-type argument will allow us to prove the asymptotic convergence of the system's trajectories to the sliding surface.

In this way, recall that $L(t)$ is a lower-bounded function with negative semidefinite derivative. Thus, $L(t)$ converges (i.e., it has finite limit when $t \rightarrow \infty$ ). Therefore, both $\Phi(t)$ and $\tilde{g}(t)$ possess finite limits as $t \quad \rightarrow$ $\infty$ since $\hat{g}(t)$ cannot be oscillatory due to (36). Now assume that $\Phi(t)$ does not converge to zero. Hence, there exist two positive constants $t_{0}$ and $c$ such that $|\Phi(t)|>c$ for $t \geq t_{0}$. Thus for $t \geq t_{0}$ (44) becomes

$$
\dot{L}(t) \leq-\eta \Gamma c<0 .
$$

The integration of (45) leads to

$$
L(t) \leq L\left(t_{0}\right)-\eta \Gamma c t
$$

implying that, asymptotically, $L(t)$ becomes negative. However, this is impossible and, therefore, the initial assumption is false and $\Phi(t)$ must converge to zero asymptotically. This fact can also be proved in an alternative manner as follows. Since $L(t)=L(\Phi(t), \widetilde{g}(t))$ is a positive definite function with negative semidefinite derivative, we have the fact that it is bounded, it has finite limit, and $0 \leq L(t) \leq L(0)<+\infty$ for all $t \geq 0$. Therefore, $0 \leq L(\infty) \leq L(0)<+\infty$. Thus, the integration of (44) yields

$$
\begin{aligned}
& L(\infty)-L(0) \leq-\eta \Gamma \int_{0}^{\infty}|\Phi(t)| d t \\
& +\infty>L(0)-L(\infty) \geq \eta \Gamma \int_{0}^{\infty}|\Phi(t)| d t \geq 0 .
\end{aligned}
$$

Since the improper integral of the right-hand side of (47) is convergent, we must have $\lim _{t \rightarrow \infty}|\Phi(t)|=0$. Hence, we can write

$$
\Phi(t)=e(t)+\lambda \int_{0}^{t} e(\tau) d \tau=\varepsilon(t)
$$

with $\varepsilon(t) \rightarrow 0$ as $t \rightarrow \infty$. Furthermore, the latter implies in the Laplace domain

$$
\lim _{t \rightarrow \infty} \varepsilon(t)=\lim _{s \rightarrow 0} s \mathscr{E}(s)=0,
$$

where $\mathscr{E}(s)$ denotes the Laplace transform of $\varepsilon(t)$. Thus, if we take the Laplace transform to (48) we obtain

$$
E(s)+\frac{\lambda}{s} E(s)=\mathscr{E}(s)
$$

since $e(0)=0$ due to the reference signal definition through (8)-(10). Therefore,

$$
E(s)=\frac{s}{s+\lambda} \mathscr{E}(s)
$$

In this way,

$$
\begin{aligned}
\lim _{t \rightarrow \infty} e(t) & =\lim _{s \rightarrow 0} s E(s)=\lim _{s \rightarrow 0} \frac{s^{2}}{s+\lambda} \mathscr{E}(s) \\
& =\lim _{s \rightarrow 0} \frac{s}{s+\lambda} \times \lim _{s \rightarrow 0} s \mathscr{E}(s)=0 \times 0=0
\end{aligned}
$$

since both limits exist and are finite. Consequently, the tracking error vanishes asymptotically. Thus, $\left(R(t)-N_{\text {ref }}(t)\right) \rightarrow$ $0,(R(t)-N(t)) \rightarrow 0$ as $t \rightarrow \infty$ and all the population becomes immune. As a consequence, the control objective of removing the illness from the population is achieved without precise a priori knowledge of the parameters of the system or of their bounds, which is a rather common situation.

In addition, this control law can also be applied to models described by any incidence rate since it does not appear explicitly in the control equations.

From a practical point of view, the discontinuous control laws (19) and (35) may cause the undesirable effect of chattering [30]. In order to avoid this phenomenon, the signum function is changed by the saturation one [34] in (19) and (35) in order to obtain a continuous control signal. Thus, the general control law (35), for instance, would read

$$
V(t)=V_{\mathrm{eq}}(t)-\frac{\widehat{g}(t)}{N(t)} \Gamma \operatorname{sat}(\Phi(t)),
$$

where sat $(\cdot)$ stands for the saturation function defined as

$$
\operatorname{sat}(\Phi)= \begin{cases}\operatorname{sgn}(\Phi) & \text { if }|\Phi| \geq \Lambda \\ \frac{\Phi}{\Lambda} & \text { if }|\Phi|<\Lambda\end{cases}
$$

with $\Lambda>0$ denoting the amplitude of the boundary layer of the saturation [34]. Furthermore, the sliding gain adaptation equation (36) must also be accordingly changed to

$$
\frac{d \widehat{g}(t)}{d t}=\Gamma \Phi(t) \operatorname{sat}(\Phi(t)), \quad \widehat{g}(0)=0
$$

These laws are usually implemented in practice and used in the numerical examples of Section 4. From a theoretical point of view, the change of the sign by the saturation implies that the tracking error (8) cannot be proved to vanish asymptotically but only to be ultimately bounded as the following theorem states.

Theorem 10. Consider the SEIR epidemic model (1)-(4) with control law (53)-(55). Thus, if Assumptions 1 and 8 hold for an unknown but finite constant upper-bound $\bar{b}$ and $\Gamma \geq 1$, then the tracking error $e(t)$ is asymptotically ultimately bounded. 
Proof. The proof is similar to that of Theorem 9. Thus, consider the Lyapunov candidate function (37). Its timederivative is given by

$$
\begin{aligned}
\dot{L}(t)= & \Phi \dot{\Phi}+\tilde{g} \dot{\hat{g}} \\
= & \Phi(t)[(\widehat{\rho}-\rho) R(t)+(\gamma-\widehat{\gamma}) I(t) \\
& \quad+(\widehat{\delta}-\delta) N(t)-\widehat{g}(t) \Gamma \operatorname{sat}(\Phi(t))] \\
& +\widetilde{g}(t) \Gamma \Phi(t) \operatorname{sat}(\Phi(t)) \\
\leq & \Phi(t)[b(x, t)-\widehat{g}(t) \Gamma \operatorname{sat}(\Phi)] \\
& +(\widehat{g}(t)-g(t)) \Gamma \Phi(t) \operatorname{sat}(\Phi) \\
= & b(x, t) \Phi(t)-\widehat{g}(t) \Gamma \Phi(t) \operatorname{sat}(\Phi) \\
& \quad-g \Gamma \Phi(t) \operatorname{sat}(\Phi)+\widehat{g} \Gamma \Phi(t) \text { sat }(\Phi) \\
= & b(x, t) \Phi(t)-g \Gamma \Phi(t) \text { sat }(\Phi) \\
\leq & |b(x, t)| \Phi(t)-\bar{b} \Gamma \Phi(t) \text { sat }(\Phi)-\eta \Gamma \Phi(t) \text { sat }(\Phi)
\end{aligned}
$$
read

Now, if $|\Phi(t)| \geq \Lambda$, then $\operatorname{sat}(\Phi(t))=\operatorname{sgn}(\Phi(t))$ and (61)

$$
\begin{aligned}
\dot{L}(t) & \leq|b(x, t)| \Phi(t)-\bar{b} \Gamma \Phi(t) \text { sat }(\Phi)-\eta \Gamma \Phi(t) \text { sat }(\Phi) \\
& =|b(x, t)| \Phi(t)-\bar{b} \Gamma|\Phi(t)|-\eta \Gamma|\Phi(t)| \\
& \leq-\eta \Gamma|\Phi(t)|<0
\end{aligned}
$$

Therefore, if $|\Phi(t)| \geq \Lambda$, then $\dot{L}(t)<0$. On the other hand, if $|\Phi(t)|<\Lambda$, then (61) reads

$$
\begin{aligned}
\dot{L}(t) & \leq|b(x, t)| \Phi(t)-\bar{b} \Gamma \Phi(t) \frac{\Phi(t)}{\Lambda}-\eta \Gamma \Phi(t) \frac{\Phi(t)}{\Lambda} \\
& \leq \bar{b} \Lambda-\bar{b} \Gamma \Phi(t) \frac{\Phi(t)}{\Lambda}-\eta \Gamma \Phi(t) \frac{\Phi(t)}{\Lambda} \\
& =\bar{b} \Lambda-\frac{(\bar{b}+\eta) \Gamma}{\Lambda}|\Phi(t)|^{2} .
\end{aligned}
$$

Equation (67) shows that, for small values of $|\Phi(t)|$, the time-derivative of the Lyapunov function may be positive implying that the Lyapunov function $L(t)$ increases. Thus, for large values of $|\Phi(t)|, L(t)$ is decreasing but, for small values of $|\Phi(t)|, L(t)$ may be increasing. Hence, we can only prove that $L(t)$ is ultimately bounded. Furthermore, from (67), it can be proved that the Lyapunov function decreases at least until

$$
|\Phi(t)|=d=\Lambda \sqrt{\frac{\bar{b}}{(\bar{b}+\eta) \Gamma}}<\Lambda
$$

since $\eta>0$ and $\Gamma \geq 1$. Consequently,

$$
\limsup _{t \rightarrow \infty}|\Phi(t)| \leq \Lambda \sqrt{\frac{\bar{b}}{(\bar{b}+\eta) \Gamma}}=o\left(\eta^{-1}\right),
$$

so that

$$
\begin{gathered}
|\Phi(t)|=\left|e(t)+\lambda \int_{0}^{t} e(\tau) d \tau\right| \leq d, \\
-d \leq e(t)+\lambda \int_{0}^{t} e(\tau) d \tau \leq d .
\end{gathered}
$$

Therefore, we can firstly consider the upper-bound in (71):

$$
e(t)+\lambda \int_{0}^{t} e(\tau) d \tau \leq d
$$

Now, introduce a function $f(t)$ with $0 \leq f(t) \leq d$ such that (71) holds with the equal sign:

$$
e(t)+\lambda \int_{0}^{t} e(\tau) d \tau=d-f(t)
$$

Equation (73) can be solved for $e(t)$ by using the Laplace transform:

$$
\begin{aligned}
& E(s)+\frac{\lambda}{s} E(s)=\frac{d}{s}-F(s), \\
& (s+\lambda) E(s)=d-s F(s), \\
& E(s)=\frac{d}{s+\lambda}-\frac{s}{s+\lambda} F(s) .
\end{aligned}
$$

The right-hand side of (76) can be rewritten as

$$
E(s)=\frac{d}{s+\lambda}-\frac{s}{s+\lambda} F(s)=\frac{d}{s+\lambda}-F(s)+\frac{\lambda}{s+\lambda} F(s) .
$$

Now, taking the inverse Laplace transform to (77), we get

$$
e(t)=\mathscr{L}^{-1}[E(s)]=d \cdot e^{-\lambda t}-f(t)+\mathscr{L}^{-1}\left[\frac{\lambda}{s+\lambda} F(s)\right] .
$$

The last term of the right-hand side of (78) is calculated as

$$
\begin{aligned}
\mathscr{L}^{-1}\left[\frac{\lambda}{s+\lambda} F(s)\right] & =\lambda \int_{0}^{t} e^{-\lambda(t-\tau)} f(\tau) d(\tau) \\
& =\lambda e^{-\lambda t} \int_{0}^{t} e^{\lambda \tau} f(\tau) d \tau \\
& \leq d e^{-\lambda t}\left(e^{\lambda t}-1\right) \\
& =d-d e^{-\lambda t} .
\end{aligned}
$$

Thus, by combining (78) and (82), we get

$$
e(t) \leq d \cdot e^{-\lambda t}-f(t)+d-d \cdot e^{-\lambda t}=d-f(t) .
$$

On the other hand, if we take the lower-bound in (71)

$$
-d \leq e(t)+\lambda \int_{0}^{t} e(\tau) d \tau
$$


we can follow the same steps as before to get

$$
e(t) \geq f(t)-d
$$

Therefore,

$$
|e(t)| \leq|d-f(t)| \leq d=\Lambda \sqrt{\frac{\bar{b}}{(\bar{b}+\eta) \Gamma}}
$$

and $e(t)$ is ultimately bounded to a residual ball of

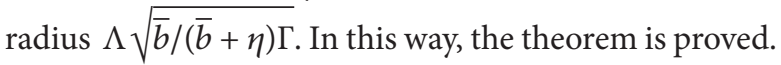

Remark 11. Notice that the radius of the convergence ball depends inversely on $\eta$. This is a positive constant used only for theoretical developments which can be selected as being arbitrary large. Thus, the radius of the convergence ball is arbitrary small implying that, from a practical viewpoint, the tracking error is also arbitrary small. Therefore, the change of the sign by the saturation does not change the tracking capabilities of the control law and it offers a good performance in practice.

\section{Simulation Examples}

This section contains some simulation examples illustrating the effectiveness of the proposed robust control laws. Since the adaptive approach presented in Section 3.1 is the most general frame, applicable to a wider range of situations, only examples with the adaptation of the sliding gain will be included. The actual parameters of the model are given by

$$
\begin{array}{r}
\mu^{-1}=255 \text { days, } \quad \sigma^{-1}=1.2 \text { days, } \\
\omega^{-1}=12 \text { days, } \quad \gamma=\sigma, \\
\nu^{-1}=115 \text { days, } \quad \beta=1.66 \text { days }^{-1}
\end{array}
$$

while the nominal parameters are given by

$$
\begin{array}{cc}
\widehat{\mu}^{-1}=215 \text { days, } & \widehat{\sigma}^{-1}=2 \text { days, } \\
\widehat{\omega}^{-1}=20 \text { days, } & \widehat{\gamma}=\widehat{\sigma}, \\
\widehat{\nu}^{-1}=150 \text { days, } \quad \widehat{\beta}=1.36 \text { days }^{-1} .
\end{array}
$$

The nominal parameters do not coincide with the actual ones. Furthermore, there is no assumption on the range of the actual parameters; that is, there is no information on their potential bounds. However, the proposed robust control law will be able to achieve the control objective regardless of these uncertainties. Also, the model's parameters are taken from an outbreak of influenza in a British boarding school in the late 1970 s, where the $\mu$ and $\nu$ parameters have been modified in such a way that a short-term simulation is enough to show the dynamic behaviour of the system. The standard incidence rate is used for simulations. The parameters of the controller are

$$
\Gamma=1, \quad \Lambda=10, \quad \lambda=\epsilon=\frac{1}{2} \text { days }^{-1} .
$$

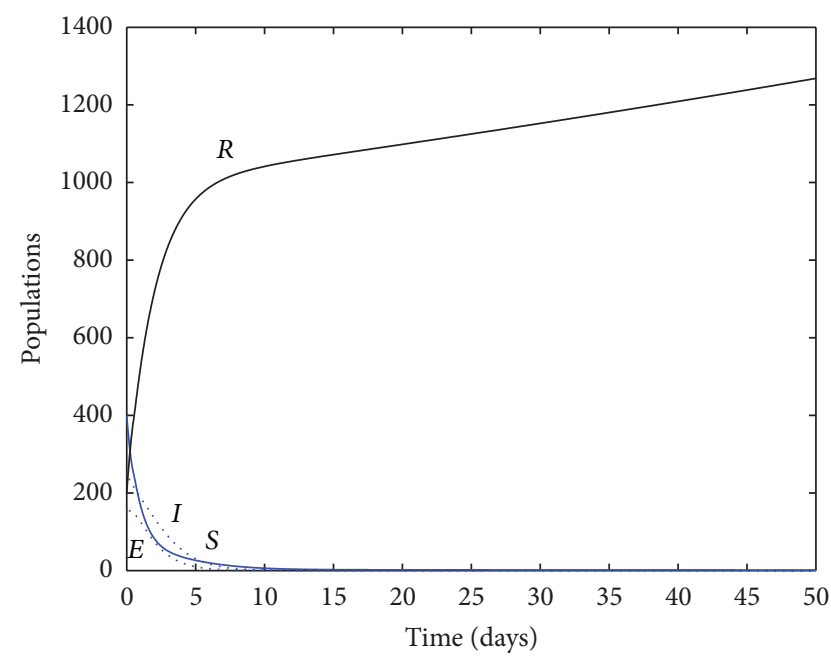

FIGURE 1: Dynamics of the system with the robust sliding-mode controller.

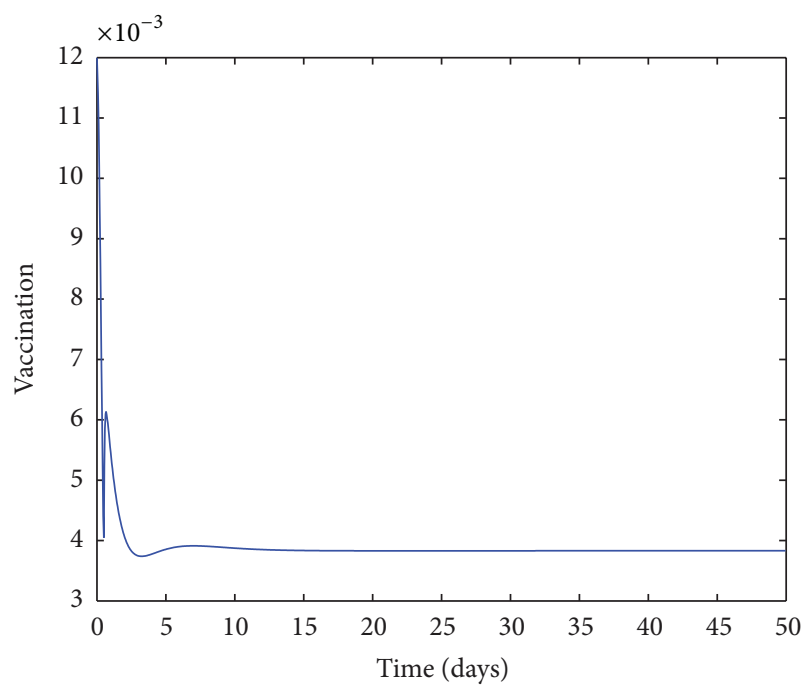

FIgURE 2: Applied vaccination control law.

With the proposed controller (53), the closed-loop dynamics of the system is depicted in Figure 1.

As can be appreciated in Figure 1, the immune population tends to be the total population while the susceptible, infective, and infectious populations vanish. This objective is attained through the vaccination function depicted in Figure 2. As expected, there is no chattering due to the utilization of the saturation function instead of the sign one. Thus, the use of the saturation makes the control action smooth and the relay effect is appreciated neither in the output nor in the vaccination function. Furthermore, it can also be appreciated that the tracking error is really small as stated in Remark 11. Moreover, the vaccination function satisfies $V(t) \in[0,1]$ as its definition imposes.

Figure 3 shows the evolution of the time-varying switching gain $\widehat{g}(t)$. It increases until the sliding condition is met and then stops. The reached value is able to make the system 


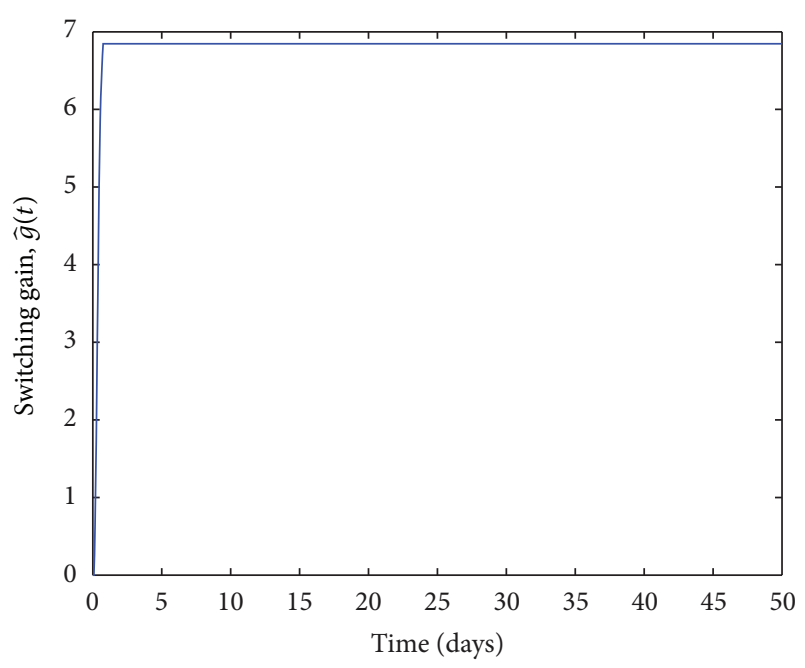

FIGURE 3: Evolution of the time-varying switching gain $\widehat{g}(t)$.

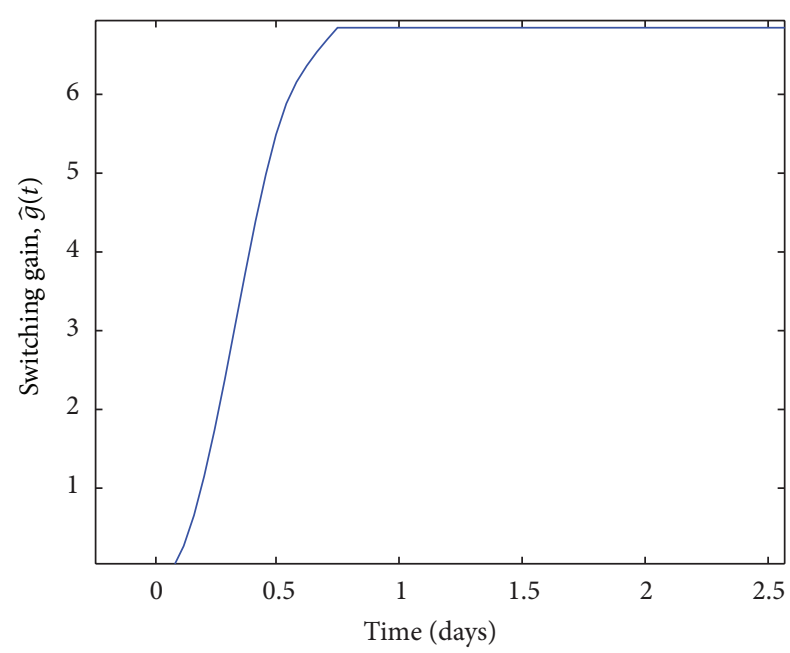

FIgURE 4: Zoom on the evolution of the switching gain $\widehat{g}(t)$.

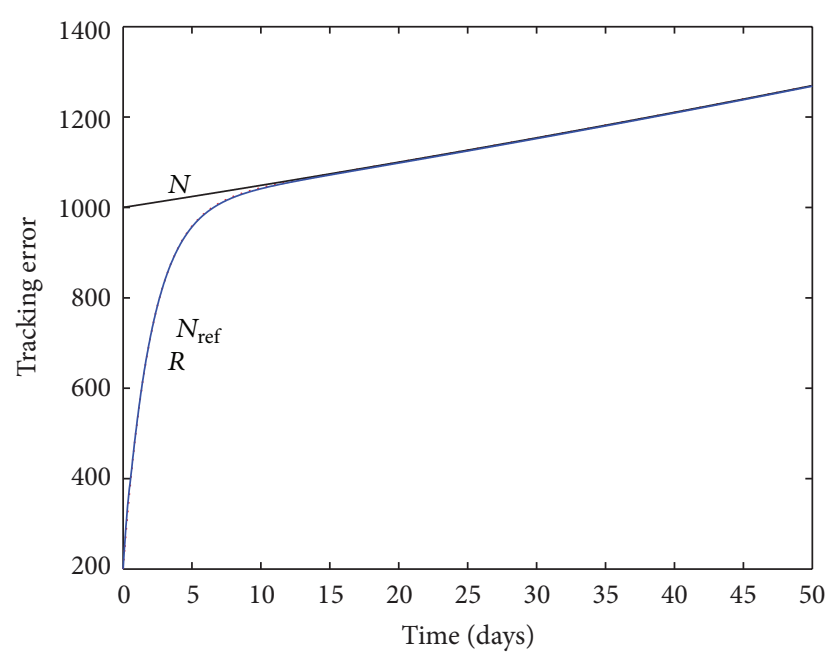

FIGURE 5: Tracking properties of the closed-loop.

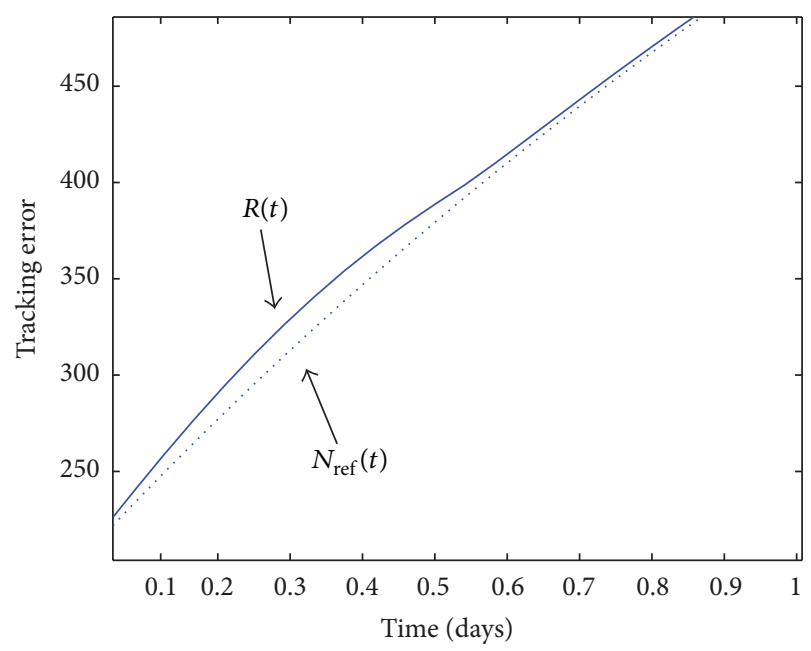

Figure 6: Zoom on the tracking properties of the closed-loop configuration.

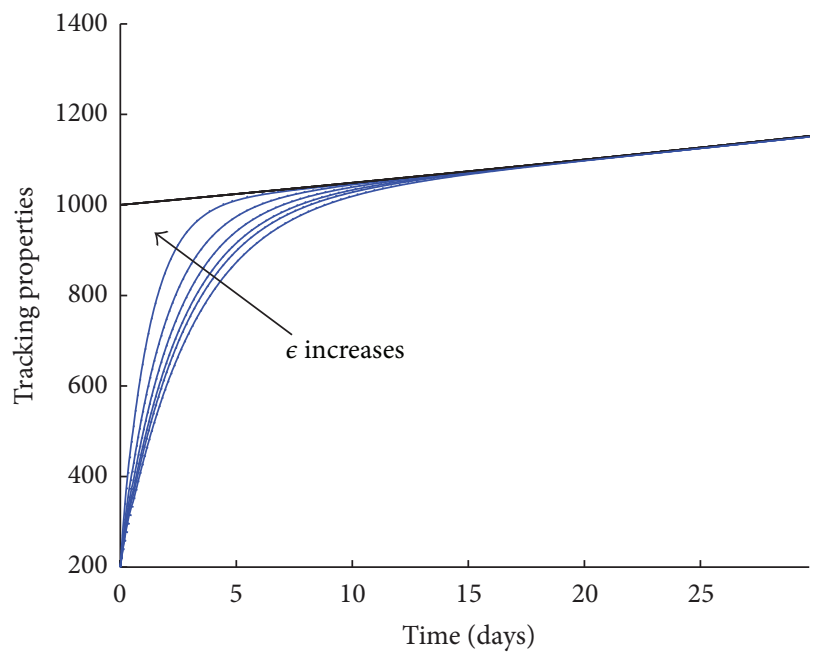

FIgURE 7: Tracking properties of the closed-loop for different values of $\epsilon$.

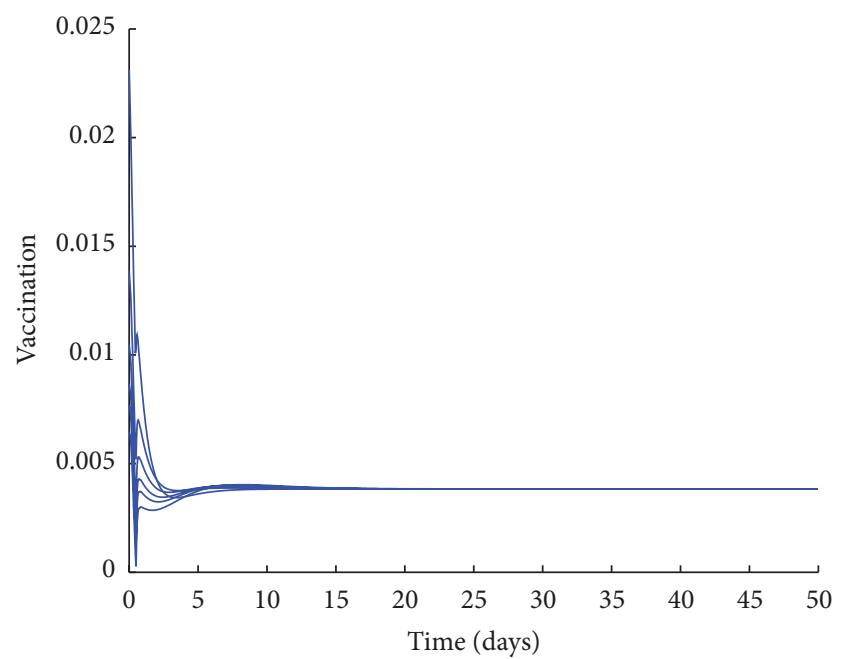

FIGURE 8: Vaccination for different values of $\epsilon$. 


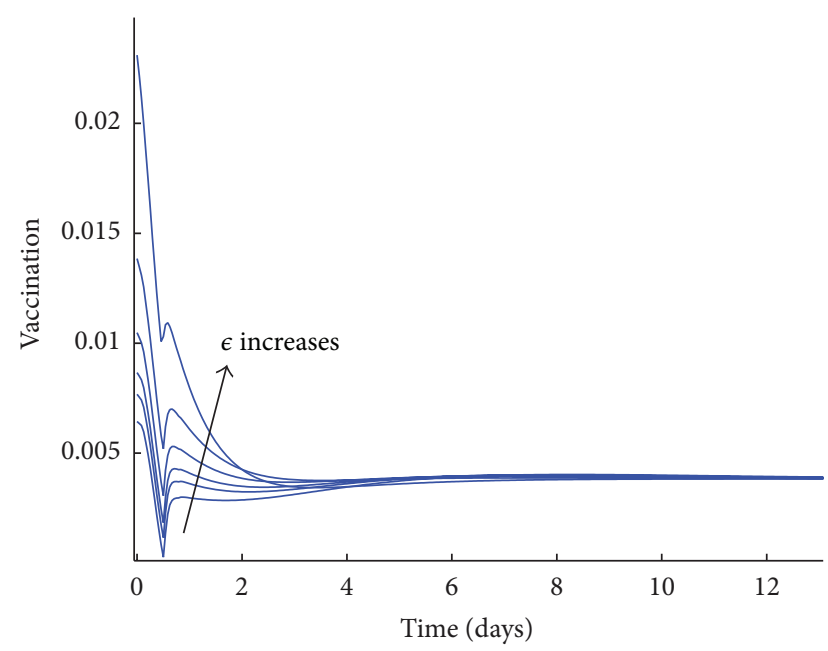

Figure 9: Zoom on the vaccination for different values of $\epsilon$.

achieve the control objective. Figure 4 shows a zoom of its evolution, especially during its increasing phase.

Finally, Figure 5 depicts the evolution of the immune population, $R(t)$, the reference signal $N_{\text {ref }}(t)$, and the total population $N(t)$. It can be appreciated that the immune population and the reference signal are practically superimposed meaning that the control objective is actually achieved. There is a slight difference between the immune population and the reference signal during the transient response as Figure 6 shows. This difference appears because of the adaptation process of the switching gain. However, this difference vanishes with time as the switching gain increases its value.

Notice that the parameter $\epsilon$ of the reference signal controls the rate at which the total population becomes immune. The greater this value is, the faster the total population will tend to become immune. The selection of this parameter influences the control action (i.e., the vaccination effort) and the closed-loop tracking shape. Since $\lambda=\epsilon$, a variation in the convergence rate changes the control law through $\lambda$. In this way, a number of simulations have been conducted for different values of $\epsilon$ (i.e., for $\lambda$ ) to illustrate the behaviour of the system for different convergence rates. Thus, Figure 7 shows the different convergence rates obtained for different values of $\epsilon$. It can be appreciated that, as $\epsilon$ increases, the time required to arrive at the total population is smaller. The control law also changes depending on the convergence rate, as Figure 8 shows. However, since all the reference signals converge to the same value, $N(t)$, then all the control laws converge to the same steady-state. The transient response is, nonetheless, different. Figure 9 shows a zoom on the different vaccination laws. As the convergence rate increases (i.e., $\epsilon$ increases), the peak value of the vaccination is larger, since a larger vaccination effort must be performed in order to attain the desired convergence rate.

Therefore, the control objective is achieved regardless of the mismatch in the parameter values and the incidence function used. In this way, the proposed sliding-mode control has been able to deal with this problem.

\section{Conclusions}

This paper applies the sliding-mode control to a SEIR epidemic model. Initially, the controller is designed assuming certain knowledge on the upper-bounding of the uncertainty signal. Then, this condition is removed while an adaptive sliding control system is designed. The proposed control law is able to achieve the control objective regardless of the parametric uncertainties of the model and the lack of a priori knowledge of the system. In this way, the infective and the infectious populations vanish and the epidemic is eradicated. Mathematical proofs of all the results stated in the paper are developed. Some numerical simulation examples complete the paper.

\section{Conflict of Interests}

The authors declare that there is no conflict of interests regarding the publication of this paper.

\section{Acknowledgments}

This work was partially supported by the Spanish Ministry of Economy and Competitiveness through Grant no. DPI201230651, the Basque Government (Gobierno Vasco) through Grant no. IE378-10, and by the University of the Basque Country through Grant no. UFI 11/07.

\section{References}

[1] M. J. Keeling and P. Rohani, Modeling Infectious Diseases in Humans and Animals, Princeton University Press, Princeton, NJ, USA, 2008.

[2] S. Zhang and Y. Zhou, "Dynamics and application of an epidemiological model for hepatitis C," Mathematical and Computer Modelling, vol. 56, no. 1-2, pp. 36-42, 2012.

[3] D. O. Gerardi and L. H. A. Monteiro, "System identification and prediction of dengue fever incidence in Rio de Janeiro," Mathematical Problems in Engineering, vol. 2011, Article ID 720304, 13 pages, 2011.

[4] F. A. C. C. Chalub and M. O. Souza, "The SIR epidemic model from a PDE point of view," Mathematical and Computer Modelling, vol. 53, no. 7-8, pp. 1568-1574, 2011.

[5] X. Zhou and J. Cui, "Stability and Hopf bifurcation analysis of an eco-epidemiological model with delay," Journal of the Franklin Institute, vol. 347, no. 9, pp. 1654-1680, 2010.

[6] Q. Gan, R. Xu, Y. Li, and R. Hu, "Travelling waves in an infectious disease model with a fixed latent period and a spatiotemporal delay," Mathematical and Computer Modelling, vol. 53, no. 5-6, pp. 814-823, 2011.

[7] N. Yi, Q. Zhang, K. Mao, D. Yang, and Q. Li, "Analysis and control of an SEIR epidemic system with nonlinear transmission rate," Mathematical and Computer Modelling, vol. 50, no. 9-10, pp. 1498-1513, 2009.

[8] X. Zhou and J. Cui, "Analysis of stability and bifurcation for an SEIR epidemic model with saturated recovery rate," Communications in Nonlinear Science and Numerical Simulation, vol. 16, no. 11, pp. 4438-4450, 2011. 
[9] M. de la Sen, R. P. Agarwal, A. Ibeas, and S. Alonso-Quesada, "On the existence of equilibrium points, boundedness, oscillating behavior and positivity of a SVEIRS epidemic model under constant and impulsive vaccination," Advances in Difference Equations, vol. 2011, Article ID 748608, 32 pages, 2011.

[10] B. Mukhopadhyay and R. Bhattacharyya, "Existence of epidemic waves in a disease transmission model with two-habitat population," International Journal of Systems Science, vol. 38, no. 9, pp. 699-707, 2007.

[11] J. Li, Y. Xiao, F. Zhang, and Y. Yang, "An algebraic approach to proving the global stability of a class of epidemic models," Nonlinear Analysis: Real World Applications, vol. 13, no. 5, pp. 2006-2016, 2012.

[12] S. Bowong and J. J. Tewa, "Global analysis of a dynamical model for transmission of tuberculosis with a general contact rate," Communications in Nonlinear Science and Numerical Simulation, vol. 15, no. 11, pp. 3621-3631, 2010.

[13] H. Chen and J. Sun, "Global stability of delay multigroup epidemic models with group mixing and nonlinear incidence rates," Applied Mathematics and Computation, vol. 218, no. 8, pp. 4391-4400, 2011.

[14] J. P. Tian and J. Wang, "Global stability for cholera epidemic models," Mathematical Biosciences, vol. 232, no. 1, pp. 31-41, 2011.

[15] H. Shu, D. Fan, and J. Wei, "Global stability of multi-group SEIR epidemic models with distributed delays and nonlinear transmission," Nonlinear Analysis: Real World Applications, vol. 13, no. 4, pp. 1581-1592, 2012.

[16] S.-Z. Huang, "A new SEIR epidemic model with applications to the theory of eradication and control of diseases, and to the calculation of $R_{0}$," Mathematical Biosciences, vol. 215, no. 1, pp. 84-104, 2008.

[17] M. de la Sen, A. Ibeas, and S. Alonso-Quesada, "On vaccination controls for the SEIR epidemic model," Communications in Nonlinear Science and Numerical Simulation, vol. 17, no. 6, pp. 2637-2658, 2012.

[18] M. de la Sen, A. Ibeas, and S. Alonso-Quesada, "Feedback linearization-based vaccination control strategies for true-mass action type SEIR epidemic models," Nonlinear Analysis: Modelling and Control, vol. 16, no. 3, pp. 283-314, 2011.

[19] S. Alonso-Quesada, M. de la Sen, R. P. Agarwal, and A. Ibeas, "Anobserver-based vaccination control law for an SEIR epidemic modelbased on feedback linearization techniques for nonlinear systems," Advances in Difference Equations, vol. 2012, p. 161, 2012.

[20] S. Gao, L. Chen, and Z. Teng, "Pulse vaccination of an SEIR epidemic model with time delay," Nonlinear Analysis: Real World Applications, vol. 9, no. 2, pp. 599-607, 2008.

[21] S. Gao, Z. Teng, and D. Xie, "The effects of pulse vaccination on SEIR model with two time delays," Applied Mathematics and Computation, vol. 201, no. 1-2, pp. 282-292, 2008.

[22] C.-Y. Chen and G. T.-C. Chiu, "Ho robust controller design of media advance systems with time domain specifications," International Journal of Innovative Computing, Information and Control, vol. 4, no. 4, pp. 813-828, 2008.

[23] S. Tong, W. Wang, and L. Qu, "Decentralized robust control for uncertain T-S fuzzy large-scale systems with time-delay," International Journal of Innovative Computing, Information and Control, vol. 3, no. 3, pp. 657-672, 2007.

[24] M. Wang, X. Liu, and P. Shi, "Adaptive neural control of purefeedback nonlinear time-delay systems via dynamic surface technique," IEEE Transactions on Systems, Man, and Cybernetics $B$, vol. 41, no. 6, pp. 1681-1692, 2011.

[25] A. Ibeas and M. de la Sen, "Robust sliding control of robotic manipulators based on a heuristic modification of the sliding gain," Journal of Intelligent and Robotic Systems, vol. 48, no. 4, pp. 485-511, 2007.

[26] C. Yang, Z. Yang, X. Huang, S. Li, and Q. Zhang, "Modeling and robust trajectory tracking control for a novel six-rotor unmanned aerial vehicle," Mathematical Problems in Engineering, vol. 2013, Article ID 673525, 13 pages, 2013.

[27] Z. Xiao-Yu, Z. Yu-Xin, X. De-Xin, and H. Kun-Peng, "Sliding mode control for mass moment aerospace vehicles using dynamic inversion approach," Mathematical Problems in Engineering, vol. 2013, Article ID 284869, 11 pages, 2013.

[28] Y.-C. Chung, B.-J. Wen, and Y.-C. Lin, "Optimal fuzzy slidingmode control for bio-microfluidic manipulation," Control Engineering Practice, vol. 15, no. 9, pp. 1093-1105, 2007.

[29] M. K. Khan and S. K. Spurgeon, "Robust MIMO water level control in interconnected twin-tanks using second order sliding mode control," Control Engineering Practice, vol. 14, no. 4, pp. 375-386, 2006.

[30] H. Lee and V. I. Utkin, "Chattering suppression methods in sliding mode control systems," Annual Reviews in Control, vol. 31, no. 2, pp. 179-188, 2007.

[31] O. Barambones and P. Alkorta, "A robust vector control for induction motor drives with an adaptive sliding-mode control law," Journal of the Franklin Institute, vol. 348, no. 2, pp. 300-314, 2011.

[32] O. Barambones, J. M. Gonzalez de Durana, and M. de la Sen, "Robust speed control for a variable speed wind turbine," International Journal of Innovative Computing, Information and Control, vol. 8, no. 11, pp. 7627-7640, 2012.

[33] A. Ibeas, M. de la Sen, and S. Alonso-Quesada, "Sliding mode robust control of SEIR epidemic models," in Proceedings of the 21st Iranian Conference on Electrical Engineering, Mashhad, Iran, May 2013.

[34] J. J. Slotine, Applied Nonlinear Control, Prentice, 1994. 


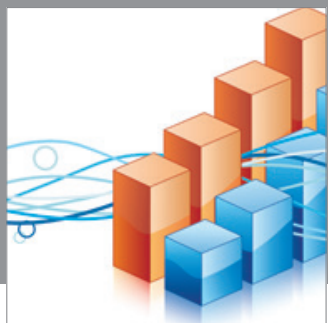

Advances in

Operations Research

mansans

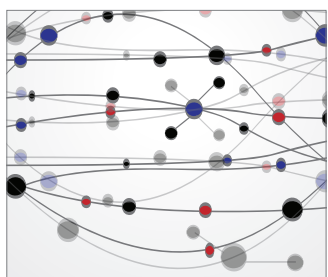

The Scientific World Journal
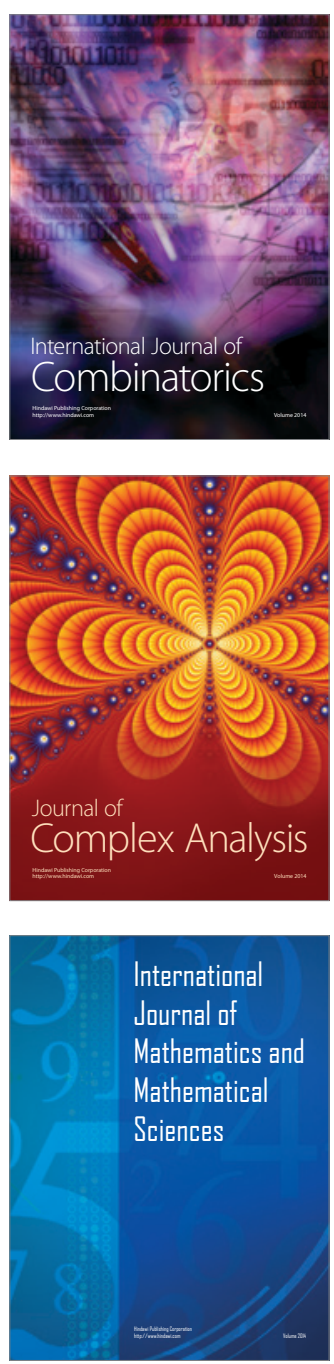
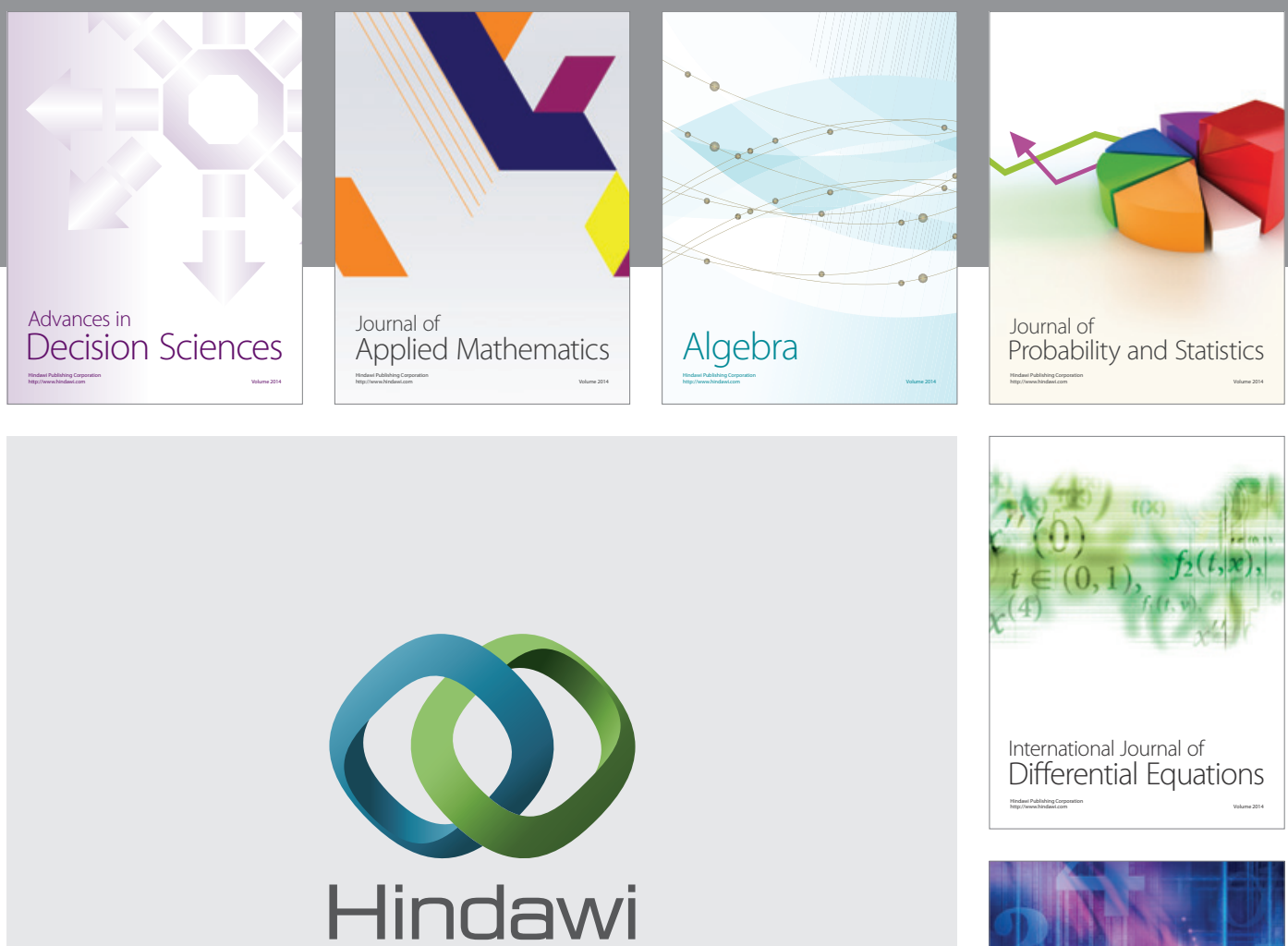

Submit your manuscripts at http://www.hindawi.com
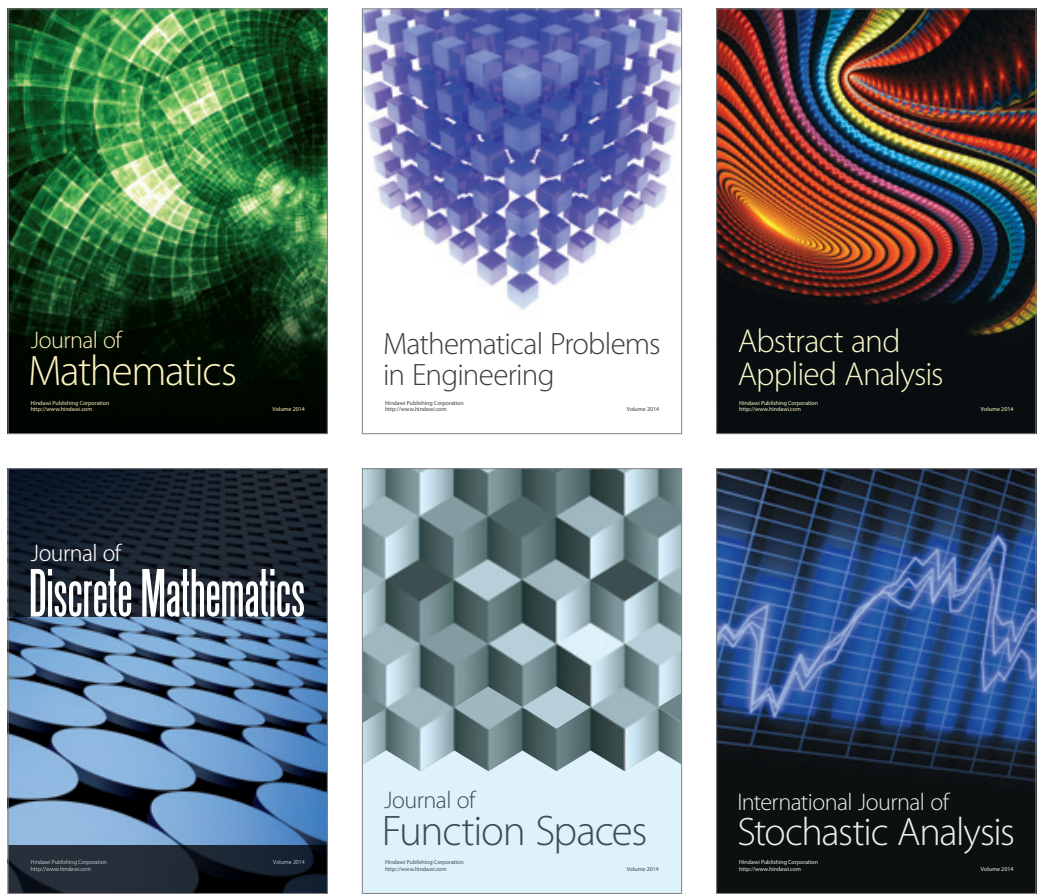

Journal of

Function Spaces

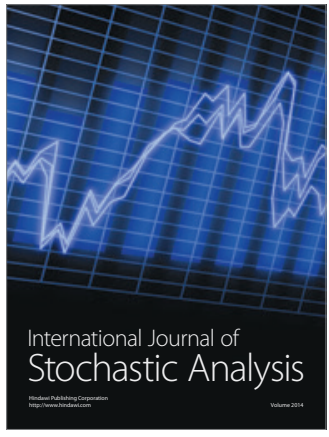

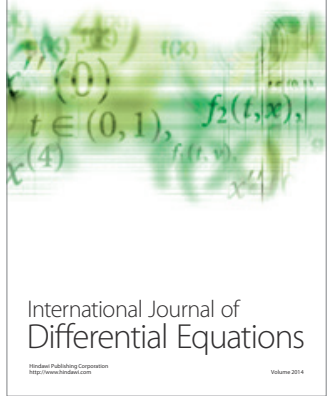
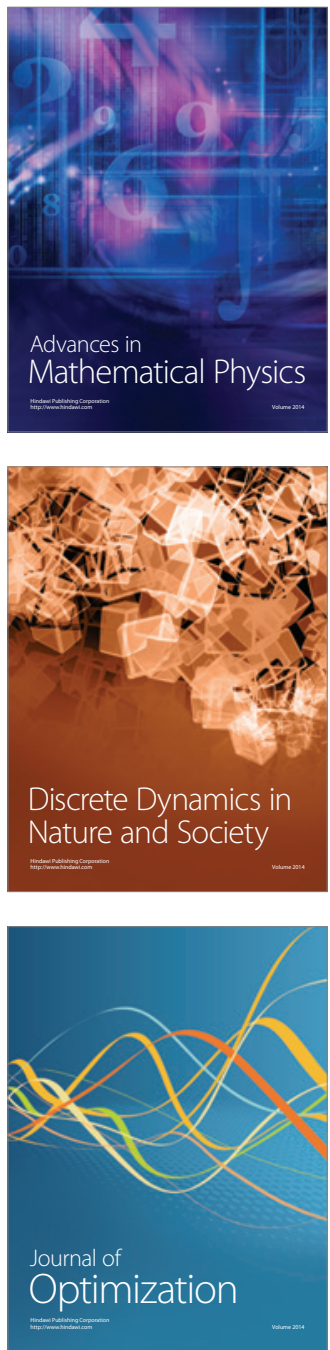